\title{
Design of an Experimental System for Wear Assessment of Slurry Pumps
}

\author{
Yao Wang, Ming J. Zuo, and Xianfeng Fan \\ Department of Mechanical Engineering, University of Alberta, Edmonton
}

\begin{abstract}
This paper discusses the design of an experimental system for assessing wear condition of slurry pumps. Several issues needed to be addressed; these include process conditions, typical wear patterns, the instrumentation and data acquisition system. This system will enable us to collect data indicating the extent to which the wetted components in a given slurry pump are worn.
\end{abstract}

Key words: Experimental System; Slurry Pump; Wear Pattern; Data Acquisition; Process Parameters; Vibration Signals.

\section{Introduction}

Slurry pumps play an important role in oil sands operations. Slurries contain abrasive and erosive solid particles, which eventually cause wear of wetted components pumps. Wear of slurry pump impellers and other wetted components is a main cause that makes pumps out of work. Due to the variability of operation parameters and slurry properties, there is a large variation in the working intervals of slurry pumps. To fully utilize the life of the wetted components in slurry pumps, there is a need to develop effective indicators of the extent to which the wetted components in a given slurry pump are worn. The University of Alberta is collaborating with industry on a research project monitoring the condition of slurry pumps in order to assess slurry pump wear.

Erosion wear was assumed to follow three mechanisms [1]: directional impact, random collisions, and Coulombic friction. The wear mechanisms were categorized as impact and scouring in [2]. Numerous investigations have been conducted to study the relationship between the wear rate of wetted components and various process conditions/slurry properties. Rayan and

\footnotetext{
* To whom correspondence should be addressed. Department of Mechanical Engineering, University of Alberta, Edmonton, Alberta, T6G 2G8, ming.zuo@ualberta.ca (e-mail), (780) 492-4466 (phone), (780) 492-2200 (fax).
}

Shawky [3] studied the erosion wear of impellers as a function of flow velocity, and concluded that the erosion wear rate was proportional to flow velocity. Through a test rig at Warman International [4], Walker et al $[5,6,7,8]$ conducted a series of experimental studies on the wear rate of key wetted components with different materials and particle sizes in different slurry pump designs. Field tests were conducted in [7, 8] and compared to laboratory studies; these confirmed that smal scale laboratory test resutls matched actual field measurement data. Water and air create a corrosive environment in slurry pumps. The combined effect of erosion and corrosion will accelerate the wear rate.

The effects of slurry on pump performance have also been studied. Through experiments, Gahlot et al [9] plotted the head/efficiency ratios versus process parameters such as particle size, concentration, and density. Additional plots between the head ratio and various process parameters were provided in [10]. According to [11], impeller wear may reduce pump head by $30 \%$ and drop pump efficiency by $15 \%$; heavily worn sideliners may reduce pump efficiency by $5 \%$.

These reported experimental studies on slurry pumps were either for improvement of their design or for predicting wear life, assuming steady state and constant operating conditions. Because of the dynamic conditions under which a pump has to operate, the reported relationships between wear rate and fixed process parameters are not very useful. To help schedule shutdowns for replacement of worn components, there is a pressing need to develop new techniques for on-line assessment of wear conditions of wetted components in slurry pumps.

We are designing an experimental system intended to provide data for both simple and advanced data analysis of the correlation between the wear status of wetted components and a number of parameters. Development of such new techniques will be based on this data analysis. Laboratory testing gives us an environment with controllable variables so that studies conducted in labs at this stage can be extended to field testing in the future. Our experimental system is a test loop which contains a slurry pump and data acquisition system among other 
components. Tests will run at different rotating speeds with controlled particle properties and slurry temperatures. Performance parameters and process variables, such as head developed, efficiency, flow rate, and motor power, will be monitored. Vibration and acoustic signals will also be monitored since they may present features of the wear status of wetted components $([12,13])$.

In this paper, we will discuss various issues that have to be addressed in order to make sure that the designed system will meet the aforementioned requirements. In Section 2, we will consider the potential for scaling our findings to field pumps when selecting the slurry pump and process conditions. In Section 3, we will define wear patterns and their combinations in terms of location and severity to reflect field wear patterns observed thus far. Selection criteria for instruments and the data acquisition (DAQ) system will be discussed in Section 4. A brief description of the whole test loop and test operating procedures will be given in Section 5 followed by a summary.

\section{Pump selection and process conditions}

\subsection{Pump selection}

The slurry pump used in this experiment must meet the following criteria:

- Geometrically similar to full-scale pumps (i.e., similar suction arrangement, similar casing arrangement, similar impeller type, etc.)

- Hydraulically similar to full-scale pumps (i.e., slurry should undergo a similar process inside the pump)

- Practical and efficient with regard to replacement of wetted components

- Having wetted components that are easy to machine

- Fitting in the design of the test skid.

Following these criteria, a Warman 3/2 CAH mechanically sealed heavy duty slurry pump has been selected for the study. The pump is a horizontal centrifugal slurry pump with a maximum allowable casing pressure of $300 \mathrm{psig}$; and it is driven by a 40HP VFD (Variable Frequency Drive) motor. From experience we have concluded that, the $3 / 2$ horizontal centrifugal slurry pump, in which the inlet diameter is 3 inches and the outlet diameter is 2 inches, is the smallest pump that retains reasonable similarity to field slurry pumps which are usually $30 / 24$. The pump will be equipped with a stainless steel liner and a stainless steel impeller to make modification of the internal profiles easier than is possible with the standard high chrome white iron.

\subsection{Process conditions}

When selecting process conditions, we should consider their similarity to full-scale plant conditions. Although some differences are inevitable, approximating full-scale conditions is the goal. We will keep the slurry temperature at $45^{\circ} \mathrm{C}$. The maximum experimental pressure developed by the pump will vary from approximately 30 to $75 \mathrm{psi}$; this is less than that under fullscale conditions, due to experimental motor power limitations. At this stage of experimentation, known and easily duplicated particle size distributions are desired to allow for repeatable testing, therefore Ottawa test sand $(50 / 70 \mathrm{mesh})$ will be used. Test slurry will be replaced periodically to minimize variation of slurry particle size due to sand erosion. A target slurry density of $1,450 \mathrm{~kg} / \mathrm{m}^{3}$ is specified as this is close to the slurry density under full-scale conditions.

With the VFD motor, the experiment will be performed at three different pump rotating speeds (1400RPM, 1800RPM, and 2200RPM). Although in full-scale oil sand applications ideally the pumping system is designed to run at its pumps' best efficiency point (BEP), the reality is that slurry pumps (operated in series) are often operated at points significantly different from their BEPs. This is primarily due to variation of slurry properties and process set points that require the pump head to be adjusted frequently. The tendency is for the flow rate to be held constant, as stable production is given precedence over efficiency. As a result, we will measure variables at fixed flow rate values which will include both the BEP and off-BEPs of each testing speed with the pump under good conditions without wear. The manufacturer's pump curve identifies the BEP for water. To find the BEPs for the test slurrywhich are expected to be different from the BEPs for water - the control valve will be adjusted from fully open to partially closed until maximum efficiency is obtained.

We do not want the occurrence of cavitation due to insufficient suction pressure because that would disturb our focus on wear faults on components. Thus, through testing, the available net positive suction head (NPSHa) will be held to a constant value greater than the required net positive suction head (NPSHr) by controlling the height of the water in the suction pressure control tank. (Refer to the setup of the test loop in Section 5). 


\section{Common wear patterns}

The useful life of a slurry pump can range from a few weeks to a few years depending on the type of slurries handled and the pump's operating parameters. In this laboratory experiment, we are not going to test the pump from its new condition to its worn condition; that would be too time-consuming. The alternative is to mimic typical wear patterns on selected wetted components at different progression levels. According to field experience and previous studies [5, 14], impellers, suction liners, and volute casings are the wetted components in which wear faults are most often seen (Fig. 1). That is why these are the three components on which individual wear damage patterns will be created.

The criteria used for determining the replacement of pump components depend mainly on how the wear process affects them. There are a number of different situations that may occur, e.g., some components may be replaced even though not completely worn. This presents great difficulty in defining an individual component's wear life. A summary of wear life definitions for different components is given in [14] (see Table 1); this helps us define different progression levels to be simulated. We will simulate a severe wear condition and a medium wear condition. Combined with the testings on good condition without wear, that will give us three measurement levels for studying wear progression.

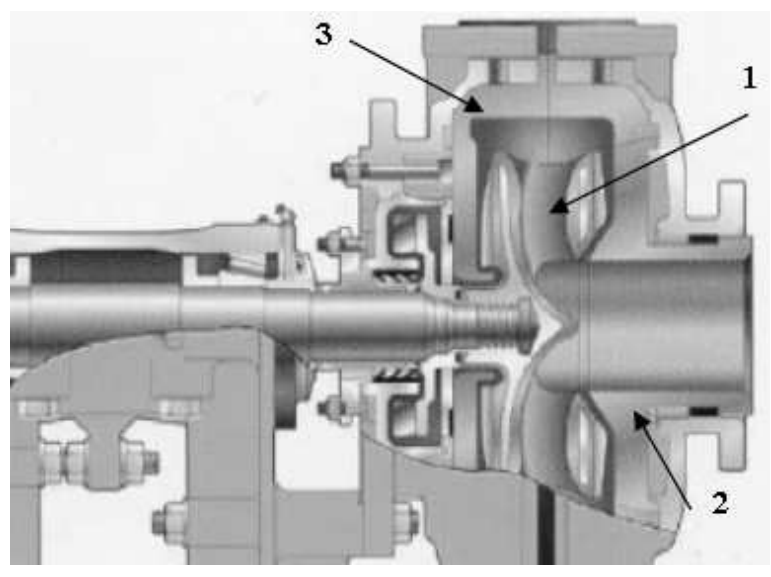

Figure 1. Wetted components in a slurry pump: 1. impeller; 2 . suction liner; 3 . volute casing.

\subsection{Individual wear patterns}

3.1.1. The wear pattern on the impeller. Two locations are found to be most easily worn [8]: the root of each vane and the area on the back shroud close to the
Table 1. Definition of wear life [14]

\begin{tabular}{|c|l|}
\hline Components & $\begin{array}{l}\text { Criteria for function failure } \\
\text { due to wear }\end{array}$ \\
\hline Impeller & $\begin{array}{l}\text { Part wear such that head developed } \\
\text { is reduced to less than the } \\
\text { process requirement, OR } \\
\text { Part wear leading to power } \\
\text { consumption that is greater } \\
\text { than available from the motor, OR } \\
\text { Uneven part wear such that the } \\
\text { vibration level is unacceptable }\end{array}$ \\
\hline Suction liner & $\begin{array}{l}\text { The component is worn through } \\
\text { to atmosphere }\end{array}$ \\
\hline Volute casing & $\begin{array}{l}\text { The component is worn through } \\
\text { to atmosphere }\end{array}$ \\
\hline
\end{tabular}

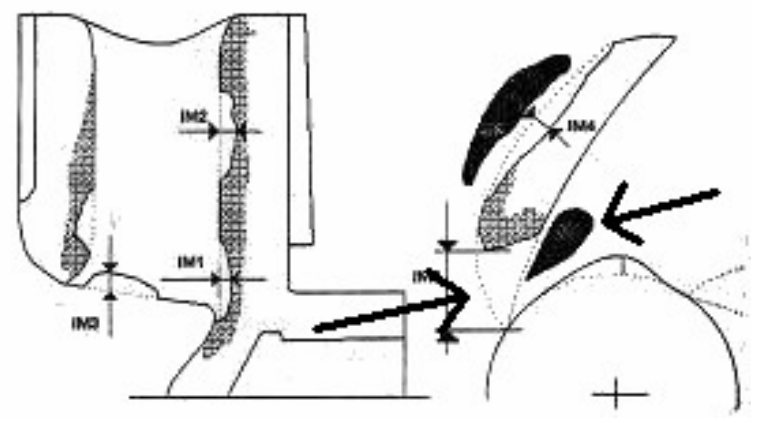

Figure 2. Locations of wear on impeller [8]

suction side of the vane root (see arrows in Fig. 2). This is confirmed by wear patterns observed in field (see Fig. 3).

For the medium wear condition level, we will mimic shallow pits on the back shroud close to the suction side of the vane root ( $50 \%$ depth of thickness of the impeller) and will remove some mass at the root of each vane (2-3\% of the impeller diameter). For the severe wear condition level, we will drill deeper pits $(90 \%$ of the depth of thickness of the impeller) and remove more mass at the roots (6-8\% of the impeller diameter).

3.1.2. The wear pattern on the suction liner. The common wear pattern found on suction liners consists of concentric or inward spiral rings on the surface facing the impeller. As the condition worsens, holes can be seen around the eye area [6, 7]. Fig. 4 shows the pattern observed in field.

For the medium wear condition level, we will mimic an inward spiral pattern spreading on the whole surface 


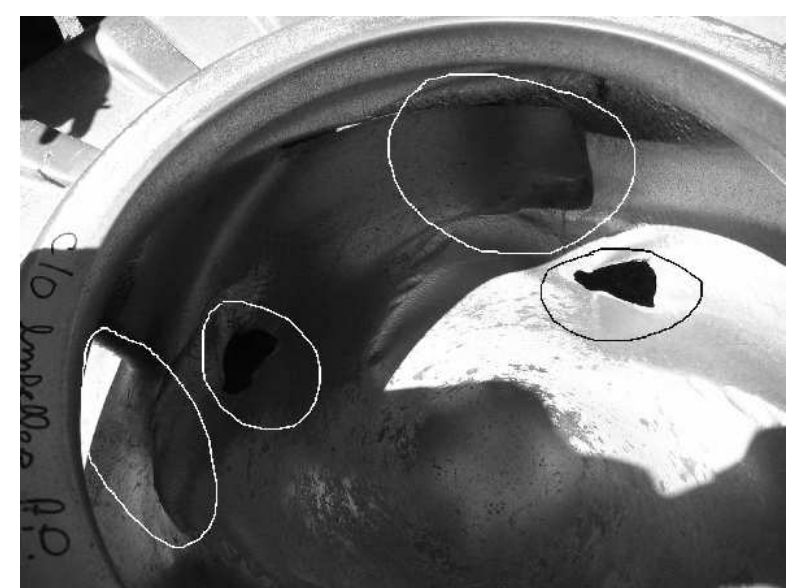

Figure 3. Impeller wear pattern observed in field operation

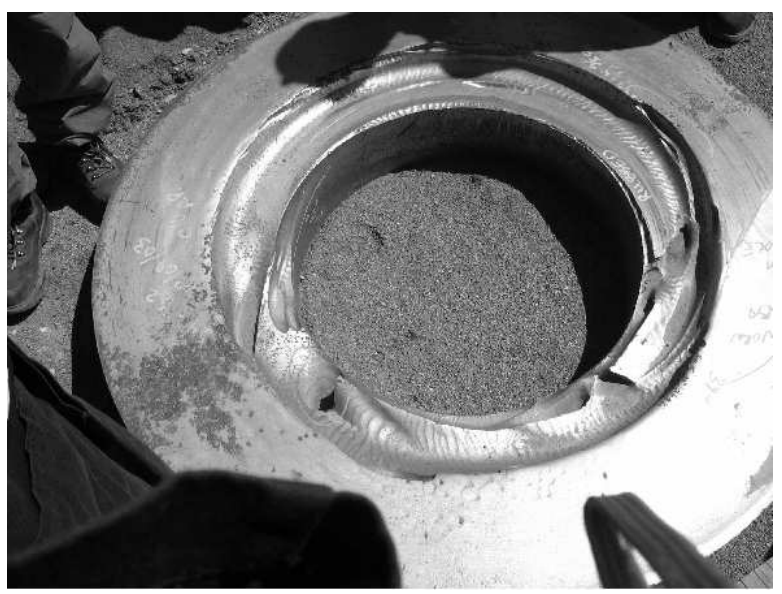

Figure 4. Suction liner wear pattern observed in field operation

of the liner (15-20 grooves, $50 \%$ of thickness of the suction liner). For the severe wear condition level, we will make the grooves deeper around the eye area, even some pits through $(90 \%$ of the thickness of the suction liner).

3.1.3. The wear pattern on the volute casing. Two common patterns are observed on volute casings [15]: wear along the side wall of the maximal radius and gouging in the wall of the cutwater (see Fig. 5).

For the selected pump, the suction liner and the casing are actually one component: a volute liner. We will create casing wear on the casing part of the volute liner. For the medium wear condition level, we will create scouring along the wall of the maximal radius $(50 \%$ of

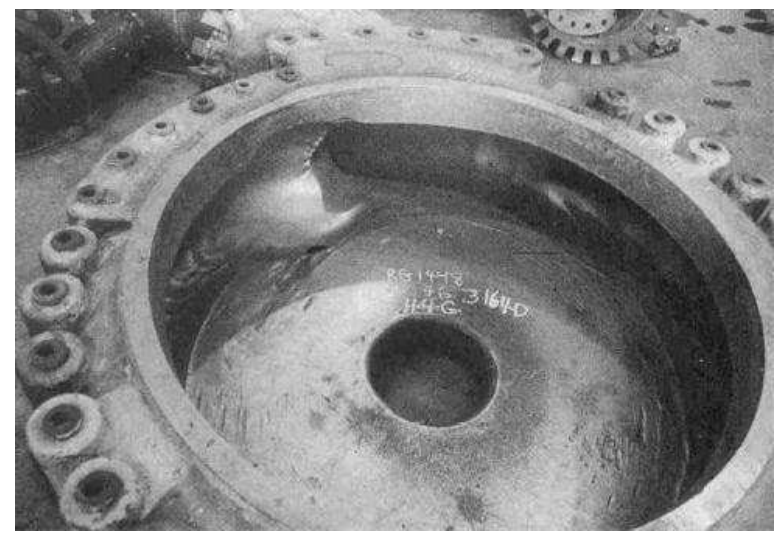

Figure 5. Typical worn pump casing ([15])

the casing thickness) and remove some mass off the cutwater (4-6\% of the casing radius). For the severe wear condition level, we will create deeper scouring $(90 \%$ of the casing thickness) and remove more mass (8-12\% of the casing radius) off the cutwater.

\subsection{Combined wear patterns}

Since in many cases wear is not seen on only one of the components, combined wear patterns will be considered as well. These combinations are selected to simulate what has been observed in field. From a summary of inspection reports on maintenance outages in field, we find that the common wear combinations are:

- impeller with slight wear + suction liner with severe wear

- impeller with severe wear + suction liner with severe wear

- impeller with severe wear + suction liner with severe wear + casing with severe wear.

After independent wear component testings, particular combinations of wear components and their progression levels will be tested using the same conditions as those of the individual wear patterns.

\section{Selection of instruments and DAQ}

\subsection{Instruments}

Instruments are used as the very front devices for monitoring and collecting physical signals. A proper selection of instruments is necessary to obtain satisfactory data sets for subsequent data analysis. Corresponding 
to the variables and signals to be measured, the selected instrumentations include accelerometers, pressure sensors, thermocouples, and a microphone.

4.1.1. Accelerometers. Accelerometers are widely used to measure vibration. They will be used in our experiment to collect vibration signals by actually monitoring the acceleration of objects. It is important to select an accelerometer with a suitable frequency response and sensitivity. The maximum rotating speed of the pump in the experiment to be conducted is approximately 2200 RPM or $36.67 \mathrm{~Hz}$. It is suspected that other periodic and transient phenomena may occur at frequencies higher than the vane pass frequency (with the 5-vane impeller design, the maximal vane pass frequency is $183.35 \mathrm{~Hz}$ ). These frequencies are a result of phenomena such as vane pass and/or slurry particle collisions with defects. Thus, we are interested in frequencies and/or harmonics that may exist at multiples of up to 10 times the fundamental frequency of $36.67 \mathrm{~Hz}$, i.e., $366.7 \mathrm{~Hz}$. According to the Nyquist Sampling Theorem, in order to avoid aliasing, the sampling frequency should be at least 2.56 times as large as the frequency of the signal to be analyzed. This, however, is only the minimum precision requirement; additional precision is desirable. Applying an additional factor of 10 times the maximum frequency of interest results in the requirement to sample at a frequency of $3667 \mathrm{~Hz}$, so the accelerometers to be used should have a frequency range larger than $3667 \mathrm{~Hz}$.

Vibration signals from three directions are to be collected at the same time so triaxial accelerometers are preferable. Two PCB Triaxial ICP (Integrated Circuit Piezoelectric) accelerometers with $100 \mathrm{mV} / \mathrm{g}$ sensitivity \& $2-5 \mathrm{kHz}$ frequency range and one PCB Triaxial ICP accelerometer with $1000 \mathrm{mV} / \mathrm{g}$ sensitivity \& 0.5-3 $\mathrm{kHz}$ range have been selected. The reason for selecting an accelerometer with a shorter range but a higher sensitivity is to monitor any vibration with relatively low amplitude that may exist in the system. One normal accelerometer and one high sensitivity accelerometer will be mounted at the pump casing near the suction of the pump (location B in Fig. 6) where it will be close to the wetted components. Another normal one will be mounted at the bearing of the shaft (location A in Fig. 6) since this location is sensitive to the vibration transmitted from the stuffing box. Flexible spools are used at both suction and discharge sides of the pump to reduce piping vibrations.

\subsubsection{Other instruments.}

Pressure sensors: The maximum discharge pressure that the pump can safely operate at is 300 psig.

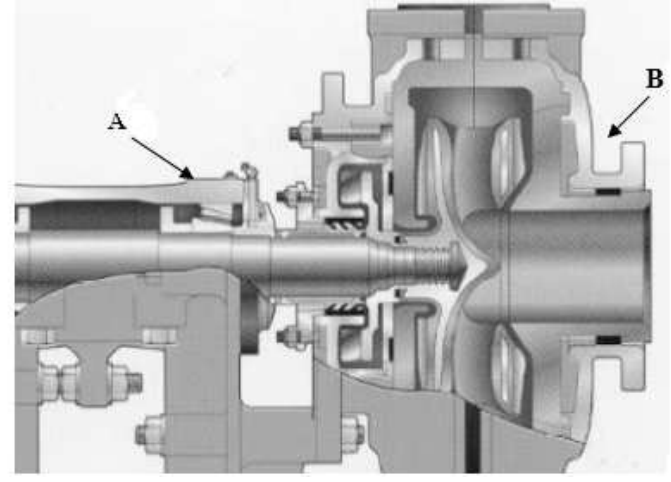

\section{Figure 6. Schematic of the locations of the ac- celerometers}

Two PCB dynamic piezoelectric sensors with 1000 psi maximal range, $5 \mathrm{mv} / \mathrm{psi}$ sensitivity and 0.01 psi resolution have been selected to be located at suction and discharge respectively. The frequency ranges of these signals' response to a worn component condition are uncertain, therefore such dynamic pressure sensors should have relatively high sensitivity, high frequency response range, and high resolution.

Thermocouples: The thermocouple that we are going to use to monitor process temperature is Omega CO1-E-20. They have a continuous temperature range from $-195 \mathrm{C}$ to $260 \mathrm{C}$. This is ample because in the experiment the slurry temperature is kept at approximately $45 \mathrm{C}$.

Microphone: The microphone used for measuring acoustic signals should have a high frequency range of up to $20 \mathrm{kHz}$. The product we have selected is a PCB prepolarized condenser microphone which has a range of 3.15-20,000 Hz.

\subsection{The data acquisition system}

Data acquisition is the process of collecting and measuring electrical signals from sensors, transducers, and other instruments, and inputting them to computers for processing. The data acquisition system is a combination of PC-based measurement hardware and software. We have selected NI LabView 7.0 as our measurement application software because it is easy to build a graphic measurement interface with the help of a large set of tools and objects. The selected hardware is provided by NI DAQ which is highly compatible with our software application. Various modules are mounted into a 12-slot SCXI chassis which has good capacity for other 
future experimental studies (see Fig. 7). Modules are selected according to the specifications of selected instrumentations. For example, the selected 4-channel ICP accelerometer module provides up to $20 \mathrm{kHz}$ of lowpass filter per channel that matches the range of the accelerometers.

The performance of all instrumentations and DAQ devices will be confirmed during the commissioning stage of the test loop in order to reaffirm our measurements.

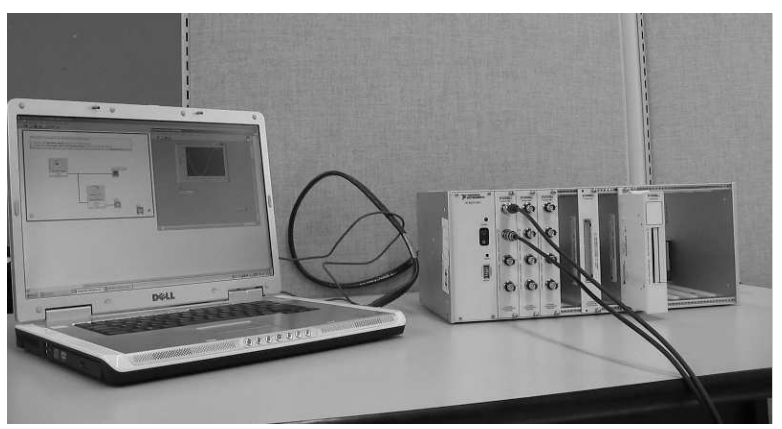

Figure 7. Data acquisition system for the slurry pump experiment

\section{Test loop and operation procedures}

Fig. 8 shows the integrated test loop which will be set up in a pilot plant. This paper covers the key issues but not all aspects of the experimental system design. Also important to the success of the experiment are other tasks such as test loop pipeline design, heat exchanger/cooling system design, and safety /environmental risk assessment. These, however, are not our focus in this paper.

After commissioning of the system, one baseline test on clear water and another baseline test on slurry will be conducted with all the pump components in good condition. All individual and combined wear patterns will be tested at three pump speeds. New sand will be substituted for old sand at the outages of replacements for different worn components. All tests are to be performed at three constant flow rates which are the BEPs in baseline testing with slurry at rotating speeds of 1400 RPM, 1800 RPM and 2200 RPM respectively, therefore for each speed we have a BEP and two off-BEPs. For baseline tests with water and slurry, although methods do exist to convert water performance to slurry performance, we will collect data at a greater number of flow rate values to confirm the manufacturer's pump curve and the performance conversion to slurry. Detailed test steps are prepared in an operation procedure document. Here we

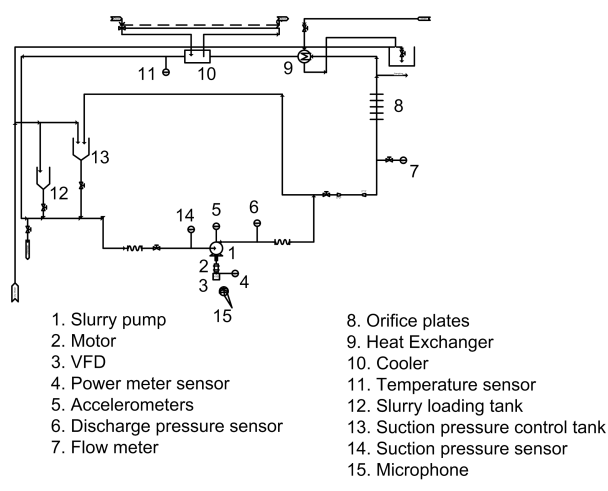

Figure 8. Schematic of the test loop

mention two points. One is the length of the data sampling duration. When the system is in steady operation, three data sets will be collected with a certain interval and a reasonable sampling duration. A relatively long sampling duration is preferred for at least one complete cycle of the lowest frequency in order to better distinguish frequencies. Because the lowest rotating speed of the pump is $1400 \mathrm{RPM}$, the lowest frequency component of the pump is $23.33 \mathrm{~Hz}$. As a result, the collection time of each data set should be longer than 0.04 seconds so that the signal in a period will appear totally in the time domain. Because there may be signals that correlate to component condition with frequencies occurring lower than the theoretical lowest frequency of $23.33 \mathrm{~Hz}$, it is wise to sample for a longer duration than the theoretical minimum. We will collect data for 60 seconds resulting in a frequency resolution of $1 / 60 \mathrm{~Hz}$.

Another point is concern regarding extraneous variables. Variables that are not controlled during a measurement but which may affect the value of the variables measured are called extraneous variables. Any variable that has a time-dependent trend could be an extraneous variable. In this experiment, the potential extraneous variables (e.g., sand trapping at worn areas, line voltage fluctuation, and atmospheric pressure) may introduce interference through a fixed order of setting individual values. Throughout the experiment, the measurement should be performed in a manner that will make such false trends unlikely, insuring they appear as random variations in the data set. For this reason, the order of rotating speeds will be randomized so they are not always in an increasing or decreasing sequence. 


\section{Summary}

This paper has presented the design of an experimental system which will be executed to further the study of slurry pump wear condition monitoring. This system is designed primarily to provide good scalability with regard to field conditions and satisfactory accuracy for subsequent analysis. Particular attention has been paid to key issues such as configuration of process conditions, simulation of typical wear patterns, selection of instruments and the data acquisition system, and experimental error consideration. We are expecting that such a well designed experimental system will help us develop indicators for wear status assessment of pump wetted components.

\section{Acknowledgment}

This study is supported by the Natural Sciences and Engineering Research Council of Canada (NSERC) and an industry partner.

\section{References}

[1] M.C. Roco and G.R. Addie, "Erosion wear in slurry pumps and pipes", Powder Technology, 1987, 50: 35-46.

[2] K.V. Pagalthivarthi and F.W. Helmly, "Application of materials wear testing to solids transport via centrifugal slurry pumps", Wear Testing of Advanced Materials, ASTM STP 1167, American Society for Testing and Materials, Philadelphia, 1992:114-126.

[3] M.A. Rayan and M. Shawky, "Evaluation of wear in a centrifugal slurry pump", Proceedings of the Institution of Mechanical Engineers. Part A, Journal of power and energy, 1989, 203: 19-23.

[4] Warman International, 1991. "Slurry testing of centrifugal pumps", Technical Bulletin, 1991, No. 12.

[5] C.I. Walker, P.J. Wells, and G.C. Bodkin, "The effect of flowrate and solid particle size on the wear of centrifugal slurry pumps", 5th International Symposium on Solid Liquid Flows, ASME FED, 1994, 189, 189-195.

[6] C.I. Walker, and G.C. Bodkin, "Empirical wear relationships for slurry pumps, Part 1: Side-liners", Wear, 2000, 242, 140-146.
[7] C.I. Walker, "Slurry pump side-liner wear: comparison of some laboratory and field results", Wear,2001, 250, 81-87.

[8] C.I. Walker and A. Roudnev, 2002. "Slurry pump impeller wear: comparison of some laboratory and field results", Proceedings of the 15th International Conference on Hydrotransport, BHR Fluid Engineering, Banff, Canada, 2002, 725-736.

[9] V.K. Gahlot, V. Seshadri, and R.C. Malhotra, "Effect of density, size distribution, and concentration of solid on the characteristics of centrifugal pumps", Journal of Fluid Engineering-Transaction of the ASME, 1992, 114: 386-389.

[10] K.A. Kazim, B. Maiti, and P. Chand, "A correlation to predict the performance characteristics of centrifugal pumps handling slurries", Proceedings of the Institution of Mechanical Engineers. Part A, Journal of power and energy, 1997, 211: 147-157.

[11] G.R Addie and A. Sellgren, "The effect of wear on the performance of centrifugal slurry pumps", Proceedings of ASME Fluids Engineering Division Summer Meeting, Washingdon, DC.,1998.

[12] F.M. Want, "Centrifugal slurry pump wear - plant experience", Proceedings of the 7th International Conference on Hydrotransport, paper H1, BHRA, Cranfield, UK, 1980.

[13] L. Wang, A.D. Hope and H. Sadek, "Vibration-based condition monitoring of pumps in the waste water industry", Insight: Non-destructive testing and condition monitoring, 2000, 42 (8), 500-503.

[14] C.I. Walker, "Slurry pump wear life uncertainty analysis", 14th International Conference on Slurry Handling and Pipeline Transportation: Hydrotransport 14, Maastricht, Holland, 1999, 663-679.

[15] K.C. Wilson, G.R. Addie, A. Sellgren and R. Clift, Slurry Transport Using Centrifugal Pumps, Blackie Academic and Professional, London, 1997. 